\title{
Recovery of antimicrobial susceptibility in methicillin- resistant Staphylococcus aureus (MRSA): a retrospective, epidemiological analysis in a secondary care hospital, Sapporo, Japan
}

\author{
Yuji Koike ${ }^{1,2}$, Hiroshi Nishiura ${ }^{\text {Corresp. 1,3 }}$ \\ ${ }^{1}$ Graduate School of Medicine, Hokkaido University, Sapporo, Hokkaido, Japan \\ 2 Department of Microbiology, JR Sapporo Hospital, Sapporo, Hokkaido, Japan \\ ${ }^{3}$ School of Public Health, Kyoto University, Kyoto, Kyoto, Japan \\ Corresponding Author: Hiroshi Nishiura \\ Email address: nishiurah@gmail.com
}

Anti-methicillin-resistant Staphylococcus aureus (MRSA) drugs are critical final options for treating MRSA infection. This study investigated the percentage of all $S$. aureus isolates that are resistant to methicillin and also MRSA susceptibility to other antimicrobial agents in the JR Sapporo Hospital inpatient service. The inpatient service MRSA percentages for Japan, Hokkaido, and JR Sapporo Hospital from 2010-19 were compared, exploring the annual rate of change in the MRSA percentage. We also investigated the antimicrobial use density (AUD) and its relationship with MRSA antimicrobial susceptibility in the JR Sapporo Hospital during 2019. The MRSA percentage in JR Sapporo Hospital was $61.5 \%$ (95\% Cl: $52.6-69.7)$ in 2010 but was only $51.6 \%$ (95\% Cl: 41.6-61.5) in 2019 , which is a $1.43 \%$ (95\% Cl: 0.42-2.43) annual decrease $(p=0.05)$. Regarding the MRSA antimicrobial susceptibility rate in JR Sapporo Hospital, the highest rates of annual increase were seen for minocycline (3.11\% [95\% Cl: $2.25-3.94$ ]) followed by fosfomycin $(2.85 \%$ [95\% Cl: 1.83-3.85]). Positive correlations with the AUD of anti-MRSA drugs were identified for susceptibility to erythromycin $(p<0.01)$, clindamycin $(p=0.002)$, and levofloxacin $(p=0.0005)$. A recovery of MRSA antimicrobial susceptibility was observed in our antibiogram dataset. Our study supports the potential for appropriate antimicrobial agent use in reviving MRSA antimicrobial susceptibility. 


\section{TITLE}

2 Recovery of antimicrobial susceptibility in methicillin-resistant Staphylococcus aureus (MRSA):

3 a retrospective, epidemiological analysis in a secondary care hospital, Sapporo, Japan

4 Short title: Recovery of MRSA antimicrobial susceptibility in Japan

5

6 Authors:

7 Yuji Koike ${ }^{1,2}$ and Hiroshi Nishiura ${ }^{1,3 *}$

8

9 Affiliations:

$10{ }^{1}$ Graduate School of Medicine, Hokkaido University, Kita 15 Jo Nishi 7 Chome, Kita-ku,

11 Sapporo-shi, Hokkaido 060-8638, Japan (YK: koike-jrsapporohosp@outlook.com; HN:

12 nishiurah@gmail.com)

13 2Department of Microbiology, JR Sapporo Hospital, Kita 3 Jo Higashi 1 Chome, Chuo-ku,

14 Sapporo-shi, Hokkaido 060-0033, Japan

$15{ }^{3}$ Kyoto University School of Public Health, Yoshidakonoecho, Sakyoku, Kyoto 606-8501, Japan

$17 *$ Correspondence to:

18 Hiroshi Nishiura

19 Kyoto University School of Public Health

20 Yoshidakonoecho, Sakyoku, Kyoto 606-8501, Japan

21 Tel: +81 75753 4456, Fax: +81 757534458

22 E-mail: nishiura.hiroshi.5r@kyoto-u.ac.jp 


\section{Abstract}

24 Anti-methicillin-resistant Staphylococcus aureus (MRSA) drugs are critical final options for

25 treating MRSA infection. This study investigated the percentage of all S. aureus isolates that are

26 resistant to methicillin and also MRSA susceptibility to other antimicrobial agents in the JR

27 Sapporo Hospital inpatient service. The inpatient service MRSA percentages for Japan,

28 Hokkaido, and JR Sapporo Hospital from 2010-19 were compared, exploring the annual rate of

29 change in the MRSA percentage. We also investigated the antimicrobial use density (AUD) and

30 its relationship with MRSA antimicrobial susceptibility in the JR Sapporo Hospital during 2019.

31 The MRSA percentage in JR Sapporo Hospital was 61.5\% (95\% CI: 52.6-69.7) in 2010 but was

32 only $51.6 \%(95 \%$ CI: $41.6-61.5)$ in 2019 , which is a $1.43 \%$ (95\% CI: $0.42-2.43)$ annual decrease

$33(p=0.05)$. Regarding the MRSA antimicrobial susceptibility rate in JR Sapporo Hospital, the

34 highest rates of annual increase were seen for minocycline (3.11\% [95\% CI: $2.25-3.94])$

35 followed by fosfomycin (2.85\% [95\% CI: 1.83-3.85]). Positive correlations with the AUD of

36 anti-MRSA drugs were identified for susceptibility to erythromycin $(p<0.01)$, clindamycin

$37(p=0.002)$, and levofloxacin $(p=0.0005)$. A recovery of MRSA antimicrobial susceptibility was

38 observed in our antibiogram dataset. Our study supports the potential for appropriate

39 antimicrobial agent use in reviving MRSA antimicrobial susceptibility.

\section{Keywords}

42 Epidemiology; Drug resistance; Anti-Bacterial Agents; Drug Utilization; Antibiogram 


\section{Introduction}

46 Staphylococcus aureus, a Gram-positive coccus, is harboured by $20 \%-40 \%$ of the population,

47 usually acting as normal flora, but also frequently infecting skin, wound, blood stream, and

48 catheters from invasive treatment (e.g. central vein). In particular, methicillin-resistant

Staphylococcus aureus (MRSA), which possesses a mecA gene associated with the production of

50 Penicillin Binding Protein 2 prime (PBP2'), is globally recognized as the most widespread

51 antimicrobial resistance problem, especially in healthcare facilities (Deresinski, 2005; Kale and

52 Dhawan, 2016; Lee et al., 2018). There has been growing concern over the spread of resistance

53 against anti-MRSA drugs in S. aureus, and the frequency of fatal outcomes in MRSA-infected

54 cases has been estimated as $64 \%$ greater than that in methicillin-sensitive S. aureus (MSSA)-

55 infected cases (World Health Organization, 2020). MRSA is epidemiologically widespread, not

56 only in Western countries but also in Asia (Chen and Huang, 2014). In July 2007, the Ministry of

57 Health, Labour and Welfare of Japan first devised a nosocomial infection surveillance system,

58 known as the Japan Nosocomial Infections Surveillance (JANIS), which provides up-to-date

59 information regarding the epidemiological situation of MRSA (Ministry of Health, Labour and

60 Welfare, 2020). As of October 2020, vancomycin-resistant S. aureus (VRSA) has yet to be

61 identified in Japan (Infectious Disease Surveillance Center, 2020).

62 MRSA is the primary cause of nosocomial infection; because the risk of death in cases of

63 blood stream infection with MRSA is known to be greater than that for similar cases of MSSA,

64 treatment is required immediately upon diagnosis (Tan et al., 2001; Hassoun et al., 2017). The

65 successful treatment of $S$. aureus is highly dependent on the choice of antimicrobial agents (van

66 Hal et al., 2012), but the antimicrobial agents that can be used is limited. Recommendations

67 regarding the appropriate use of antimicrobial agents are available (Ministry of Health, Labour 
68 and Welfare, 2016; Abubakar and Sulaiman, 2018), and anti-MRSA drugs are regarded as

69 critical final options for treating MRSA infection (Chen et al., 2019). While an increase in

70 antimicrobial-resistant strains of Gram-negative rods (e.g. fluoroquinolone resistance in

71 Escherichia coli (Terahara and Nishiura, 2019)) has emerged as a significant health issue in

72 Japan, MRSA is another pressing problem with a high prevalence. Japan formulated and

73 announced the 2020 antimicrobial resistance (AMR) action plans in recognition of the 2016

74 Global Action Plan that was approved by the World Health Assembly in May 2015.

75 Accordingly, appropriate antimicrobial agent use was promoted, aiming to reduce the percentage

76 of S. aureus that are MRSA from $48.5 \%$ in 2015 to less than $20 \%$ by 2020 (Ministry of Health,

77 Labour and Welfare, 2016).

78 In Japan, when a causative agent has yet to be identified during the treatment of a

79 bacterial infection, the use of an antibiogram from the applicable regional healthcare facility is

80 recommended to design a suitable empiric therapy (Niki et al., 2017). Despite the frequent use of

81 antibiograms for determining treatment options, the associated dataset has yet to be widely used

82 for epidemiological studies (Kim et al., 2020). At JR Sapporo Hospital, a private secondary care

83 hospital in the capital city Sapporo of the northernmost prefecture, Japan, the infection control

84 team was founded in 1987, and since 2005, it has involved physicians, nurses, pharmacists, and

85 clinical laboratory technologists. There have been very few infection control doctors (ICD) who

86 truly act as infection disease specialists in Japan (Kishida and Nishiura, 2020), and the ICDs of

87 JR Sapporo Hospital have been concurrently covered by expert physicians in other specialty

88 areas (e.g. a cardiologist and renal physician, as of October 2020). To address its limitation in

89 specialty knowledge, the ICT has involved an infection control nurse since 2014, an infection

90 control microbiological technologist (ICMT) since 2017, and an infectious disease chemotherapy 
91 pharmacist since 2018. The team has continuously assessed the antimicrobial susceptibility of

92 bloodstream infections and drug-resistant bacterial agents upon identification, providing regular

93 surveillance information to each member of the hospital team. In addition, broad-spectrum

94 antibacterial agents and anti-MRSA drugs are managed on a notification basis. The impact of the

95 ICT activities on promoting appropriate antimicrobial agent use for inpatient treatment has yet to

96 be explicitly assessed.

97 The present study aimed to investigate the percentage of $S$. aureus isolates caused by

98 MRSA, the antimicrobial susceptibility of MRSA isolates, and the relationship between AUD

99 and the antimicrobial susceptibility of MRSA in the JR Sapporo Hospital inpatient service. Here,

100 we comprehensively analyse the dataset from 2010-19.

101 Materials \& Methods

102 Study setting

103 Hokkaido is the second largest island of Japan, located in the northernmost part, and Sapporo

104 city is its capital city, populated by approximately 1.9 million people. JR Sapporo Hospital is

105 within a walking distance from the main station, Sapporo station, and the total inpatient capacity

106 for providing secondary acute care is 312 beds. Additionally, the hospital is designated as the

107 Hokkaido Affiliated Hospital for Cancer Treatment; it also contributes to the primary healthcare

108 service for the region, reserving an independent ward for primary healthcare.

109 Data source at JR Sapporo Hospital

110 We aimed to analyse the percentage of $S$. aureus involving MRSA, the time-dependent changes

111 in the percentage of MRSA resistant to specific antimicrobial agents, and the time-dependent 
112 changes in the use frequency of various antimicrobial agents. From 2010-19, JR Sapporo

113 Hospital collected antimicrobial susceptibility data, which was summarized as the Weekly

114 Report for internal use (e.g. during ICT meetings or printed in the Weekly Laboratory

115 Surveillance). The present study used these published secondary data.

116 Antibiogram data, classified by antimicrobial agent and patient service (i.e. inpatient or

117 outpatient), were collected. As control data, we also extracted the openly accessible data from

118 JANIS (Ministry of Health, Labour and Welfare, 2020), which has conducted a nationwide

119 laboratory-based surveillance of antimicrobial resistance since 2000. As of 2019, a total of 2,100

120 healthcare facilities with 200 or more beds have been registered with JANIS (Ministry of Health,

121 Labour and Welfare, 2020). Regarding the JANIS data, we retrieved data from not only the

122 entire country but also from just the Hokkaido region to use for comparisons. For both the JR

123 Sapporo Hospital and JANIS datasets, the antimicrobial susceptibility records did not specify

124 whether the patient was a carrier or was actively infected; additionally, no record of sampling

125 part of the body (i.e. infection location) was available. Here, we focused on inpatient data

126 because the impact of ICT activities should be better reflected by the inpatient data than by

127 outpatient data. The applied definition of MRSA adhered to the standardized criteria published

128 by the Clinical \& Laboratory Standards Institute (CLSI), M100-S22. In JR Sapporo hospital, we

129 have consistently defined MRSA as resistant to oxacillin (MPIPC) $\geq 4 \mu \mathrm{g} / \mathrm{mL}$ or Cefoxitin (CFX)

$130 \geq 8 \mu \mathrm{g} / \mathrm{mL}$ during minimal inhibitory concentration (MIC) susceptibility testing throughout the

131 entire period of our study.

132 AUD was expressed as defined as the daily dose (DDD) per 1,000 patient-days. The basic

133 definition of DDD is the assumed average maintenance dose per day for a drug used for its main

134 indication in adults. Consequently, the AUD was calculated as (total antimicrobial 
$135 \mathrm{dose}) /(\mathrm{DDD} \times$ monthly number of inpatients $) \times 1,000$ (Lee et al., 2007; Yoshida et al., 2013). The

136 annual number of inpatients was recorded by the accounting section of the hospital.

\section{Statistical analysis}

138 Three different analyses were conducted. First, we examined the time-dependent changes in the 139 antimicrobial resistance rate of $S$. aureus over the 10-year period from 2010-19. We measured

140 the resistance rate as the percentage of all $S$. aureus isolates that are resistant to methicillin. To

141 detect trends in the percentage of antimicrobial-resistant S. aureus, we devised two simple

142 models, i.e. models with or without a time-dependent linear component, and then employed the

143 likelihood ratio test so that the presence of a trend can be identified by the significant

144 improvement in the likelihood following the incorporation of the trend into the model.

145 Second, the antimicrobial susceptibility rate of MRSA over time was examined; this was

146 similarly measured as the percentage of MRSA that were also resistant to other antimicrobial

147 agents. For calculating the $95 \%$ confidence interval (CI) of the percentage, we employed the

148 Agresti's score confidence interval because the sample sizes were not necessarily large and the

149 susceptibility rates frequently had values close to $0 \%$ or $100 \%$. The presence of a time-dependent

150 trend was also judged by a likelihood ratio test.

151 Third, we examined the relationship between the AUD and the MRSA antimicrobial

152 susceptibility rate by clinical department, so that we could investigate if the use of anti-MRSA

153 drugs is associated with the antimicrobial susceptibility rate. The relationship between these two

154 continuous variables was examined by linear correlation testing, and $p$-values of less than 0.05

155 were regarded as significant. All statistical analyses were conducted by using JMP ver 14.0.0

156 (SAS Institute Inc. Cary, NC, USA). 


\section{Ethical considerations}

158 The present study was based on a secondary dataset in which all patient information was

159 deidentified. Therefore, the present study did not require informed consent. This study was

160 approved by the institutional review board of the Hokkaido University Graduate School of

161 Medicine (Med 20-021).

\section{Data sharing}

163 In the present study, we retrospectively analysed two different datasets. The dataset from the

164 JANIS database can be retrieved online (Ministry of Health, Labour and Welfare, 2020;

165 https://janis.mhlw.go.jp/report/kensa_prefectures.html). The other dataset containing the AUD

166 by department and antimicrobial susceptibility in methicillin-resistant Staphylococcus aureus

167 (MRSA) by department in JR Sapporo Hospital, 2019 are uploaded as Supplementary Tables S1 168 and S2.

\section{Results}

170 Table 1 shows the annual trends in the number of MRSA isolates and their proportion among $S$.

171 aureus isolates across Japan, for the Hokkaido region, and in JR Sapporo Hospital. In 2019,

$17248.1 \%$ (95\% CI: 47.9-48.3) and 42.6\% (95\% CI: 41.9-43.3) of S. aureus isolates across Japan

173 and in Hokkaido, respectively, were MRSA, and the annual decline in the MRSA rate across

174 Japan was 0.94\% (95\% CI: 0.92-0.96; $p<0.01)$. For JR Sapporo Hospital, 61.5\% (95\% CI:

175 52.6-69.7) of S. aureus isolates were MRSA in 2010, and this amount declined to 51.6\% $(95 \%$

176 CI: 41.6-61.5) in 2019, corresponding to a 1.43\% (95\% CI: $0.42-2.43)$ decrease per year $(p=$

177 0.05). Although the overall proportion of MRSA in JR Sapporo Hospital was greater than that 
178 across Japan or in Hokkaido, JR Sapporo Hospital exhibited a significant decrease in its MRSA

179 rate over the ten-year period.

180

The antimicrobial susceptibility rates for MRSA in JR Sapporo Hospital and across Japan

181 are summarized in Figures 1 and 2, respectively. In JR Sapporo Hospital, the MRSA

182 susceptibility rates for arbekacin and trimethoprim-sulfamethoxazole remained substantial (close

183 to $100 \%$ ), whereas the proportions of MRSA sensitive to gentamicin, minocycline,

184 erythromycin, clindamycin, levofloxacin, and fosfomycin in 2019 were all higher than their 2010

185 levels of approximately less than $20 \%$ (Figure 1). The estimated rates of increase are shown in

186 Table 2. The highest rates of annual increase were observed for minocycline followed by

187 fosfomycin, which had annual recovery rates 3.11\% (95\% CI: 2.25-3.94) and 2.85\% (95\% CI:

188 1.83-3.85), respectively. Additionally, a significant increasing susceptibility trend was observed

189 for all drugs except for arbekacin, levofloxacin, and trimethoprim-sulfamethoxazole. The

190 susceptibility of MRSA to six antimicrobial agents has been consistently examined across Japan

191 (Figure 2), and significant increases in MRSA susceptibility (all $p<0.01$ ) were observed for

192 gentamicin, minocycline, and clindamycin, with estimated annual increase rates of $2.51 \%(95 \%$

193 CI: $1.45-3.55), 4.01 \%$ (95\% CI: 2.98-5.01), and 4.78\% (95\% CI: 3.80-5.71), respectively (Table

194 2).

195 Supplementary Tables S1 and S2 show the AUD of different antimicrobial agents and the

196 antimicrobial susceptibility of MRSA by department. The AUD of anti-MRSA drugs at JR

197 Sapporo Hospital in 2019 was highest in the dermatology department (42.8) followed by the

198 thoracic surgery (8.6), surgery (7.4), urology (6.3), orthopaedics (4.3), internal medicine (4.0),

199 and cardiology (3.6) departments. A bivariate comparison between the AUD and MRSA

200 antimicrobial susceptibility by department identified significant positive correlations for 
201 erythromycin $(p<0.01)$, clindamycin $(p=0.002)$, and levofloxacin $(p=0.0005)$ (Figure 3$)$.

202 Thus, when anti-MRSA drugs were appropriately used where MRSA was detected, the

203 antimicrobial susceptibility of MRSA was maintained at a high level.

\section{Discussion}

205 The present study examined the trend in the MRSA proportion among S. aureus isolates in

206 inpatient services across Japan, in Hokkaido, and in JR Sapporo Hospital by comparing the

207 annual rate of change in this proportion, and it also investigated the AUD and its relationship

208 with the MRSA antimicrobial susceptibility within JR Sapporo Hospital. Overall, the data exhibit

209 a clear decreasing trend in the proportion of MRSA among S. aureus isolates in Japan. Although

210 the rate of change in MRSA in Hokkaido was not significant, a marked decreased in the MRSA

211 proportion was observed in JR Sapporo Hospital over the 10-year study period. During this time,

212 there was not only an overall decline in the proportion of MRSA, but also a recovery in the

213 antimicrobial susceptibility of MRSA. Across Japan, there was an improvement in the MRSA

214 susceptibility to gentamycin, minocycline, and clindamycin, and JR Sapporo Hospital enjoyed a

215 significant recovery of MRSA susceptibility to gentamicin, minocycline, erythromycin,

216 clindamycin, and fosfomycin. Exploring the relevance of these findings to antimicrobial agent

217 use revealed that greater susceptibility to erythromycin, clindamycin, and levofloxacin was

218 found in departments where anti-MRSA drugs were more frequently used.

219 An important finding of the present study is that our antibiogram dataset exhibited not

220 only a decreasing proportion of MRSA over the study period, but also a recovery of MRSA

221 antimicrobial susceptibility, which might have been associated with the launches of ICT in 2014

222 and the antimicrobial stewardship team (AST) activities in 2017. ICT and AST activities

223 imposed a notification system for the use of anti-MRSA drugs; additionally, broad-spectrum 
224 antibacterial agents were gradually avoided and appropriate anti-MRSA drugs were used when

225 necessary. The observed recovery in MRSA susceptibility was seen for antimicrobial agents that

226 are not generally the first choice for treating MRSA; however, the overall improvements

227 observed following implementation of an antibiogram for MRSA are encouraging, particularly

228 for cases of refractory infection and complications resulting from mixed infection, because the

229 increased number of drug options enable the possibility of combination therapy and second-

230 choice antimicrobial agents (especially, when the gold standard treatment using anti-MRSA

231 drugs is not available) to be considered (Rodvold and McConeghy 2014).

232 Another notable finding is the identification of a positive correlation between the AUD

233 for anti-MRSA drugs and MRSA antimicrobial susceptibility, which highlights the critical

234 importance of maintaining antimicrobial stewardship. JR Sapporo Hospital has promoted the

235 appropriate use of antimicrobial agents since the founding of ICT, even in the absence of an

236 infectious disease specialist. In 2017, the AST introduced a new notification system for broad-

237 spectrum antimicrobial agents and anti-MRSA agents. Despite such a useful system, it is

238 generally a difficult task for clinical laboratory technologists to intervene in physician decisions

239 regarding the choice of antimicrobial agents (Roque et al., 2014), and physicians retain the right

240 to make a final decision about prescriptions. Thus, to encourage antimicrobial stewardship in the

241 hospital, infection control nurses, clinical laboratory technologists, and pharmacists have

242 strategically built their own professional training plans, obtaining expert accreditation associated

243 with the stewardship program, and made a special effort to find case-by-case solutions in

244 multiple clinical departments. Presently, such effort has been set as an essential prerequisite for

245 joining the AST in our hospital. Notably, whenever microbiological testing results were

246 submitted to physicians, clinical laboratory technologists added a short comment, which 
247 sometimes even contained explicit clinical interpretations of the testing result. Furthermore, a

248 proposed choice of antimicrobial agents for treatment was also presented, following a

249 preliminary discussion with the infection disease chemotherapy pharmacist and ward-based

250 pharmacist.

Decreases in $S$. aureus antimicrobial resistance rates have been reported in many regions

252 worldwide, including the USA, Latin America, Canada, and Europe, although the observed rate

253 of resistance varies widely by country (Chang et al., 2015; Acree et al., 2017; Arias et al., 2017;

254 Kistler et al., 2019; Nichol et al., 2019; Walter et al., 2017). Like these other countries, Japan has

255 shown a decreasing trend in the rate of MRSA. However, although the AMR Action Plan in

256 Japan aimed to achieve a decrease in the antimicrobial resistance rate of $S$. aureus from $48.5 \%$ in

2572015 to less than $20 \%$ by 2020 , the observed 2019 rate was $48.1 \%$ across Japan, $42.6 \%$ in

258 Hokkaido, and 51.6\% in JR Sapporo Hospital, values all well above the target. An important

259 learning point from our analysis is that the goal of antimicrobial resistance rate should be

260 determined using a trend analysis as shown in this study; additionally, the possibility of

261 combination therapy can also be considered to further improve antimicrobial susceptibility and

262 lower the economic cost (Rodvold and McConeghy, 2014; Davis et al., 2015; Tong et al., 2016).

263 Four technical limitations of this work must be noted. First, the present study applied a

264 descriptive analysis, with some statistical modelling support; thus, the causal effect of each

265 single treatment on antimicrobial susceptibility has not been fully demonstrated. A controlled

266 study using individual datasets should be conducted to address this gap. Second, we analysed

267 AUD only by clinical department, because that stratification was the only available information;

268 however, a similar analysis could be more clinically relevant if the data were classified by

269 diagnosed disease or clinical specimen type. Third, our analysis of the time trend was not able to 
270 incorporate age, sex, or treatment history because this information was not integrated with the

271 existing dataset. Consequently, our analysis focused purely on the temporal component alone.

272 Fourth, because of widespread policies regarding appropriate antimicrobial agent use

273 implemented since 2016, bacterial culture testing was recommended, which may have decreased

274 empiric therapy overall. Thus, the proportion of MSSA could have slightly increased from 275 around 2016.

276 While antimicrobial susceptibility testing at an individual level is critical in determining

277 the appropriate choice of antimicrobial agent(s) for successful treatment, it is also vital to analyse

278 the corresponding epidemiological data, so that the temporal dynamics of AMR can be explicitly

279 analysed and evaluated as part of risk assessment practice. The present study identified a

280 recovery of antimicrobial susceptibility to some antimicrobial drugs in MRSA in Japan and

281 supports the potential for appropriate antimicrobial agent use in reviving antimicrobial

282 susceptibility in MRSA.

283

284 Funding

285 H.N. received funding from Health and Labor Sciences Research Grants (19HA1003, 286 20CA2024, and 20HA2007), the Japan Agency for Medical Research and Development 287 (JP19fk0108104, JP20fk0108140 and JP20fk0108535s0101), the Japan Society for the

288 Promotion of Science KAKENHI (17H04701 and 21H03198), the Inamori Foundation, the Japan 289 Science and Technology Agency CREST program (JPMJCR1413); and the SICORP (e-ASIA) 290 program (JPMJSC20U3). This study was also supported by German Federal Ministry of Health 291 (BMG) COVID-19 Research and Development funding to the World Health Organization. The 
292 funders played no role in the study design, data collection and analysis, decision to publish, or

293 preparation of the manuscript.

294 Acknowledgments

295 We thank Katie Oakley, PhD, from Edanz Group (https://en-author-services.edanzgroup.com/ac)

296 for editing a draft of this manuscript.

297

298 References

299 Abubakar U, Sulaiman SAS. Prevalence, trend and antimicrobial susceptibility of Methicillin

300 Resistant Staphylococcus aureus in Nigeria: a systematic review. J Infect Public Health

301 2018;11:763-70. DOI: 10.1016/j.jiph.2018.05.013

302

303

304

305

306

307

308

309

310

311

312

313
Acree ME, Morgan E, David MZ. S. aureus Infections in Chicago, 2006-2014: Increase in CA MSSA and Decrease in MRSA Incidence. Infect Control Hosp Epidemiol 2017;38:1226-

34. DOI : 10.1017 / ice. 2017.177

Arias CA, Reyes J, Carvajal LP, Rincon S, Diaz L, Panesso D, Ibarra G, Rios R, Munita JM, Salles MJ, Alvarez-Moreno C, Labarca J, Garcia C, Luna CM, Mejia-Villatoro C, Zurita J, Guzman-Blanco M, Rodriguez-Noriega E, Narechania A, Rojas LJ, Planet PJ, Weinstock GM, Gotuzzo E, Seas C. A prospective cohort multicenter study of molecular epidemiology and phylogenomics of Staphylococcus aureus bacteremia in nine Latin American countries. Antimicrob Agents Chemother 2017;61:e00816-17. DOI : 10.1128 / AAC.00816-17

Chang VS, Dhaliwal DK, Raju L, Kowalski RP. Antibiotic resistance in the treatment of Staphylococcus aureus keratitis: a 20-year review. Cornea 2015;34:698-703. DOI : 10.1097 / ICO.0000000000000431 
314 Chen CJ, Huang YC. New epidemiology of Staphylococcus aureus infection in Asia. Clin

315 Microbiol Infect 2014;20:605-23. DOI: 10.1111/1469-0691.12705

316 Chen X, Lou W, Liu J, Ding B, Fan W, Hong J. A novel antimicrobial polymer efficiently treats

317 multidrug-resistant MRSA-induced bloodstream infection. Biosci Rep 2019;39-50. DOI:

$318 \quad 10.1042 / B S R 20192354$

319 Davis JS, Van Hal S, Tong SY. Combination antibiotic treatment of serious methicillin-resistant 320 Staphylococcus aureus infections. Semin Respir Crit Care Med 2015;36:3-16. DOI:

321 $10.1055 / \mathrm{s}-0034-1396906$

322 Deresinski S. Methicillin-resistant Staphylococcus aureus: an evolutionary, epidemiologic, and therapeutic odyssey. Clin Infect Dis 2005;40:562-73. DOI: 10.1086/427701

324

325

326

327 328

329

330

331

332 333 334 335

336

Hassoun A, Linden PK, Friedman B. Incidence, prevalence, and management of MRSA bacteremia across patient populations-a review of recent developments in MRSA management and treatment. Crit Care 2017;21:211. DOI: 10.1186/s13054-017-1801-3

IDSC Infectious Disease Surveillance Center. https://idsc.nih.go.jp/disease/vrsa/guide01.html (accessed 2 October 2020).

Kale P, Dhawan B. The changing face of community-acquired methicillin-resistant Staphylococcus aureus. Indian J Med Microbiol 2016;34:275-85. DOI: 10.4103/02550857.188313

Kim S, Yoo SJ, Chang J. Importance of Susceptibility Rate of 'the First' Isolate: Evidence of Real-World Data. Medicina 2020;56:E507. DOI: 10.3390/medicina56100507.

Kishida N, Nishiura H. Accelerating reductions in antimicrobial resistance: Evaluating the effectiveness of an intervention program implemented by an infectious disease consultant. Int J Infect Dis 2020;93:175-81. DOI: 10.1016/j.jiid.2020.01.051 
337 Kistler JM, Thoder JJ, Ilyas AM. MRSA incidence and antibiotic trends in urban hand

338 infections: A 10-year longitudinal study. Hand (N Y) 2019;14:449-54. DOI:

339 $10.1177 / 1558944717750921$

340 Lee AS, de Lencastre H, Garau J, Kluytmans J, Malhotra-Kumar S, Peschel A, Harbarth S.

341 Methicillin-resistant Staphylococcus aureus. Nat Rev Dis Primers 2018;4:18033. DOI:

$342 \quad 10.1038 /$ nrdp.2018.33

343 Lee, S. S., H. S. Kim, H. J. Kang, J. K. Kim \& D. R. Chung (2007) Rapid spread of methicillin-

344 resistant Staphylococcus aureus in a new hospital in the broad-spectrum antibiotic era. J

345 Infect, 55, 358-62. DOI: 10.1016/j.jinf.2007.06.011

346 Nichol KA, Adam HJ, Golding GR, Lagacé-Wiens PRS, Karlowsky JA, Hoban DJ, Zhanel GG;

347 Canadian Antimicrobial Resistance Alliance (CARA) and CANWARD. Characterization of

$348 \quad$ MRSA in Canada from 2007 to 2016. J Antimicrob Chemother 2019;74:iv55-63. DOI:

$349 \quad 10.1093 / \mathrm{jac} / \mathrm{dkz} 288$

350 Niki Y, Kaku M, Aoki Y, Kawaguchi T, Kosaka N, Seki M. Guidance for implementing an

$351 \quad$ antimicrobial stewardship program in Japan. Japanese Society for Infection prevention and

$352 \quad$ Control 2017, Vol32, No5.

353 http://www.kankyokansen.org/uploads/uploads/files/jsipc/ASP_guidance.pdf (accessed 2

$354 \quad$ October 2020).

355 Ministry of Health, Labour and Welfare. Japan Nosocomial Infections Surveillance (JANIS)

356 About JANIS. https://janis.mhlw.go.jp/report/kensa_prefectures.html (accessed 2 October 357 2020). 
358 Ministry of Health, Labour and Welfare. Japan Nosocomial Infections Surveillance (JANIS) List

359 of participating medical institutions. https://janis.mhlw.go.jp/hospitallist/index.html

$360 \quad$ (accessed 2 October 2020).

361 Ministry of Health, Labour and Welfare. National action plan on antimicrobial resistance (AMR)

362 2016-2020. https://www.mhlw.go.jp/file/06-Seisakujouhou-10900000-Kenkoukyoku/

363 0000138942.pdf, 2016 (accessed 2 October 2020).

364 Rodvold KA, McConeghy KW. Methicillin-resistant Staphylococcus aureus therapy: past,

365 present, and future. Clin Infect Dis 2014;58 Suppl 1:S20-7.

366 Roque F, Herdeiro MT, Soares S, Teixeira Rodrigues A, Breitenfeld L, Figueiras A. Educational

367 interventions to improve prescription and dispensing of antibiotics: a systematic review.

$368 \quad$ BMC Public Health 2014;14:1276. DOI: 10.1186/1471-2458-14-1276

369 Tan TY, Corden S, Barnes R, Cookson B. Rapid identification of methicillin-resistant

370 Staphylococcus aureus from positive blood cultures by real-time fluorescence PCR. J Clin

$371 \quad$ Microbiol 2001;39:4529-31. DOI: 10.1128/JCM.39.12.4529-4531.2001

372 Terahara F, Nishiura H. Fluoroquinolone consumption and Escherichia coli resistance in Japan:

373 an ecological study. BMC Public Health 2019;19:426. DOI: 10.1186/s12889-019-6804-3

374 Tong SY, Nelson J, Paterson DL, Fowler VG, Howden BP, Cheng AC, Chatfield M, Lipman J,

375 Van Hal S, O'Sullivan M, Robinson JO, Yahav D, Lye D, Davis JS; CAMERA2 study

376 group and the Australasian Society for Infectious Diseases Clinical Research Network.

377 CAMERA2 - combination antibiotic therapy for methicillin-resistant Staphylococcus aureus

378 infection: study protocol for a randomised controlled trial. Trials 2016;17:170. DOI:

379 $10.1186 / \mathrm{s} 13063-016-1295-3$ 
380 van Hal SJ, Jensen SO, Vaska VL, Espedido BADL, Gosbell IB. Predictors of mortality in

381 Staphylococcus aureus Bacteremia. Clin Microbiol Rev 2012;25:362-86. DOI:

$382 \quad 10.1128 /$ CMR.05022-11

383 Walter J, Noll I, Feig M, Weiss B, Claus H, Werner G, Eckmanns T, Hermes J, Abu Sin M.

384 Decline in the proportion of methicillin resistance among Staphylococcus aureus isolates

385 from non-invasive samples and in outpatient settings, and changes in the co-resistance

386 profiles: an analysis of data collected within the Antimicrobial Resistance Surveillance

387 Network, Germany 2010 to 2015 . BMC Infect Dis 2017;17:169. DOI:10.1186/s12879-017-

$388 \quad 2271-6$

389 Yoshida, J., T. Kikuchi, N. Matsubara, I. Asano \& N. Ogami (2013) Association between ward-

390 specific antimicrobial use density and methicillin-resistant Staphylococcu aureus

391 surveillance: a 60-month study. Infect Drug Resist, 6, 59-66. DOI: 10.2147/IDR.S45843

392 World Health Organization. WHO Antimicrobial Resistance. https://www.who.int/news-

$393 \mathrm{room} /$ fact-sheets/detail/antimicrobial-resistance (accessed 20 Sep 2020).

394 


\section{PeerJ}


398 Figure legends

399 Figure 1. Time-dependent changes in the antimicrobial susceptibility of methicillin400 resistant Staphylococcus aureus (MRSA) in JR Sapporo Hospital from 2010-19.

401 Percentage of MRSA isolates susceptible to various antimicrobial agents in JR Sapporo Hospital 402 from 2010-19. The yearly estimate (dot) shows the percentage of susceptible cases. The 403 whiskers cover the 95\% confidence intervals, which were calculated using Agresti's score 404 confidence interval. The MRSA susceptibility rates to vancomycin, teicoplanin, and linezolid 405 were all $100 \%$ for the period from $2010-19$.

406

407 Figure 2. Time-dependent changes in the antimicrobial susceptibility of methicillin408 resistant Staphylococcus aureus (MRSA) across Japan from 2010-19.

409 Percentage of MRSA isolates susceptible to various antimicrobial agents in JR Sapporo Hospital 410 from 2010-19. The yearly estimate (dot) shows the percentage of susceptible cases. The

411 whiskers cover the 95\% confidence intervals, which were calculated using Agresti's score 412 confidence interval, but they are mostly invisible because of the large sample size of the Japan 413 nosocomial infections surveillance (JANIS) dataset. The susceptibility rates of MRSA to 414 arbekacin and fosfomycin could not be calculated because JANIS did not include these drugs as 415 part of their routine monitoring. The MRSA susceptibility rates to vancomycin, teicoplanin, and 416 linezolid are not shown because the MRSA isolates in JR Sapporo Hospital, which is the 417 comparison group, had 100\% susceptibility to these drugs.

418
Figure 3. Bivariate correlation analysis between the AUD and the antimicrobial susceptibility rate of methicillin-resistant Staphylococcus aureus (MRSA) by ward in JR Sapporo Hospital, 2019. 
422 The correlation between the AUD and MRSA antimicrobial susceptibility rate across different

423 inpatient wards was explored. Where MRSA was present, it is likely that anti-MRSA drugs were

424 used. The antimicrobial susceptibilities of MRSA to erythromycin, clindamycin, and

425 levofloxacin were significantly correlated with AUD. GM: Gentamicin, ABK: Arbekacin,

426 MINO: Minocycline, EM: Erythromycin, CLDM: Clindamycin, LVFX: Levofloxacin, FOM:

427 Fosfomycin, ST: Trimethoprim-sulfamethoxazole, NS: not significant.

428

429 


\section{Figure 1}

Figure 1. Time-dependent changes in the antimicrobial susceptibility of methicillinresistant Staphylococcus aureus (MRSA) in JR Sapporo Hospital from 2010-19.

Percentage of MRSA isolates susceptible to various antimicrobial agents in JR Sapporo Hospital from 2010-19. The yearly estimate (dot) shows the percentage of susceptible cases. The whiskers cover the 95\% confidence intervals, which were calculated using Agresti's score confidence interval. The MRSA susceptibility rates to vancomycin, teicoplanin, and linezolid were all $100 \%$ for the period from 2010-19. 

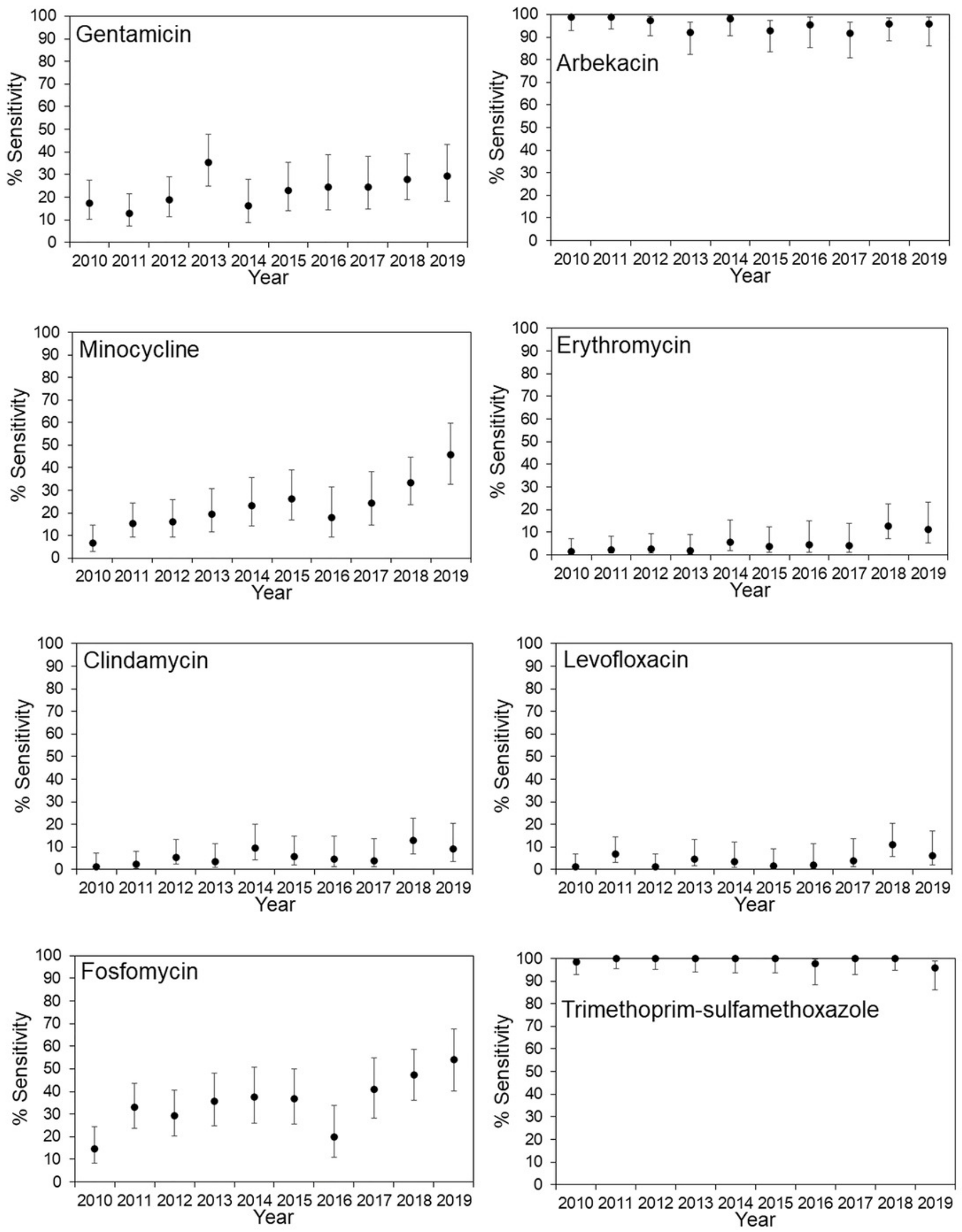


\section{Figure 2}

Figure 2. Time-dependent changes in the antimicrobial susceptibility of methicillinresistant Staphylococcus aureus (MRSA) across Japan from 2010-19.

Percentage of MRSA isolates susceptible to various antimicrobial agents in JR Sapporo Hospital from 2010-19. The yearly estimate (dot) shows the percentage of susceptible cases. The whiskers cover the 95\% confidence intervals, which were calculated using Agresti's score confidence interval, but they are mostly invisible because of the large sample size of the Japan nosocomial infections surveillance (JANIS) dataset. The susceptibility rates of MRSA to arbekacin and fosfomycin could not be calculated because JANIS did not include these drugs as part of their routine monitoring. The MRSA susceptibility rates to vancomycin, teicoplanin, and linezolid are not shown because the MRSA isolates in JR Sapporo Hospital, which is the comparison group, had $100 \%$ susceptibility to these drugs. 

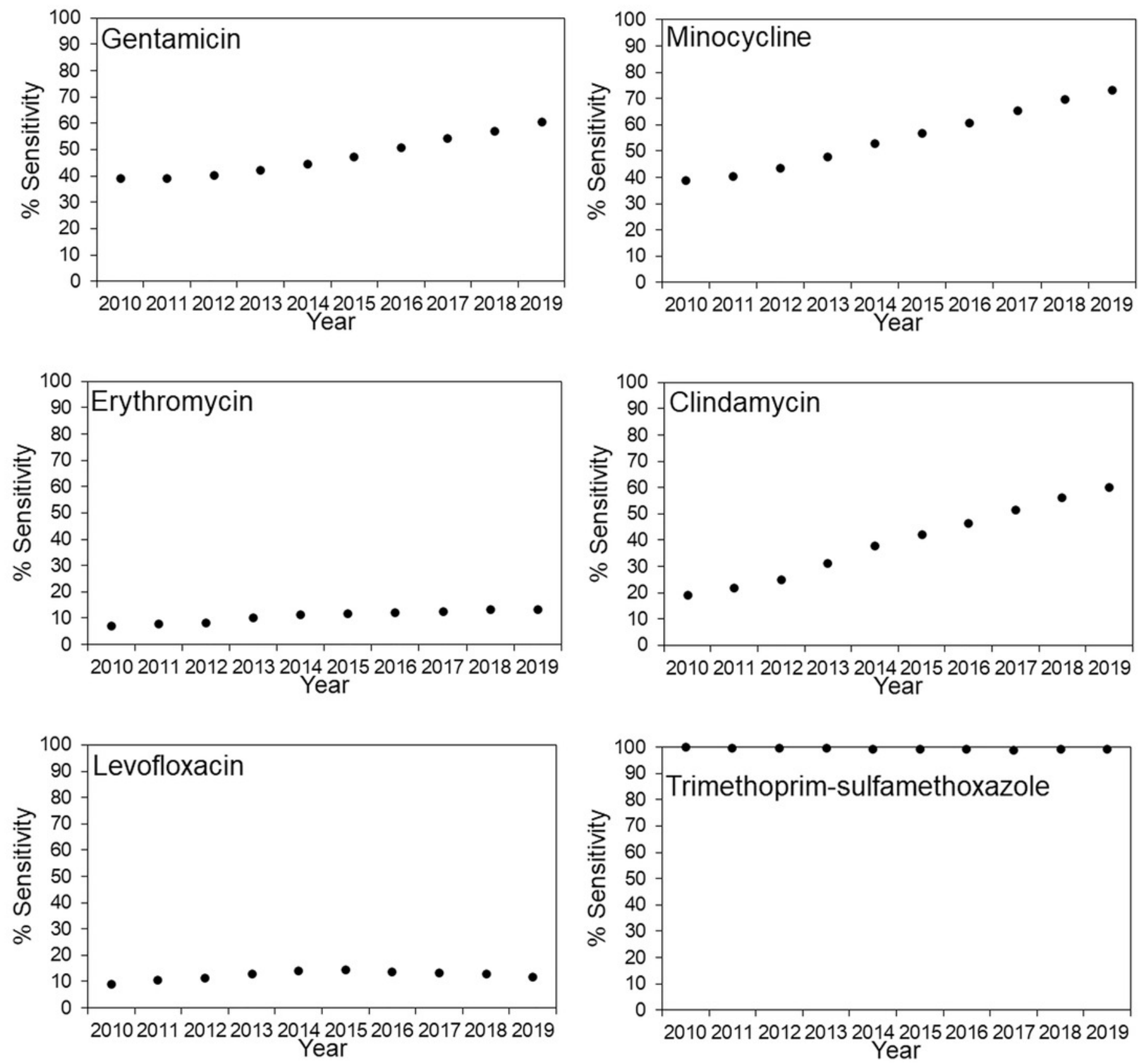


\section{Figure 3}

Figure 3. Bivariate correlation analysis between the AUD and the antimicrobial susceptibility rate of methicillin-resistant Staphylococcus aureus (MRSA) by ward in JR Sapporo Hospital, 2019.

The correlation between the AUD and MRSA antimicrobial susceptibility rate across different inpatient wards was explored. Where MRSA was present, it is likely that anti-MRSA drugs were used. The antimicrobial susceptibilities of MRSA to erythromycin, clindamycin, and levofloxacin were significantly correlated with AUD. GM: Gentamicin, ABK: Arbekacin, MINO: Minocycline, EM: Erythromycin, CLDM: Clindamycin, LVFX: Levofloxacin, FOM: Fosfomycin, ST: Trimethoprim-sulfamethoxazole, NS: not significant.

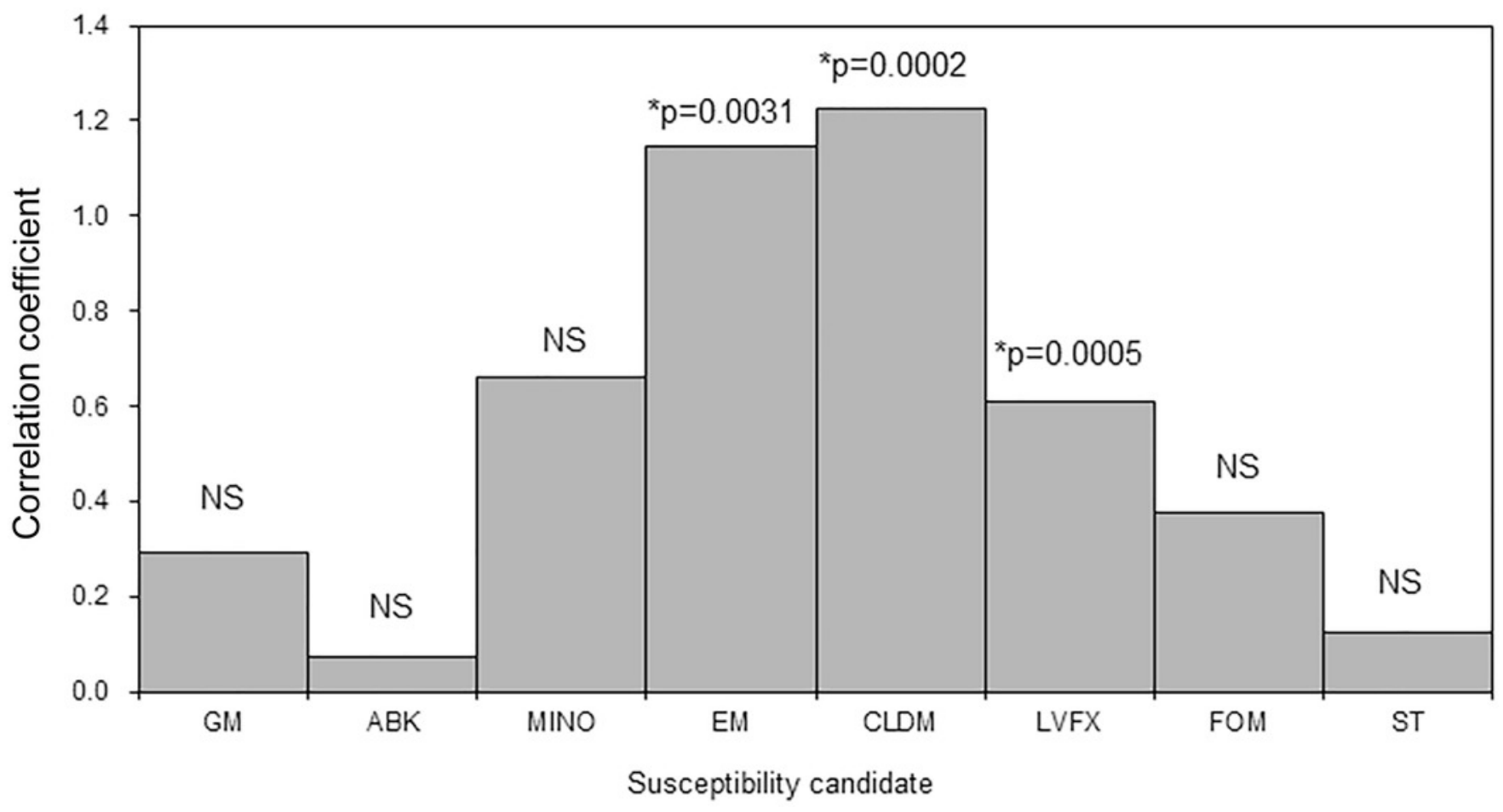




\section{Table $\mathbf{1}$ (on next page)}

Table 1. Percentages of methicillin-resistant Staphylococcus aureus (MRSA) for Japan, the Hokkaido region, and JR Sapporo Hospital from 2010-19.

The total numbers of $S$. aureus and MRSA isolates and the percentage of $S$. aureus isolates due to MRSA from 2010-19. The annual percent change (the yearly rate of decline) in the percentage of $S$. aureus isolates due to MRSA; the $p$-value indicates whether the timedependent change is statistically significant. All datasets were from inpatient records. 
1 Table 1. Percentages of methicillin-resistant Staphylococcus aureus (MRSA) for Japan, the Hokkaido region, and JR Sapporo

\section{Hospital from 2010-19.}

\begin{tabular}{|c|c|c|c|c|c|c|c|c|c|c|c|c|c|}
\hline$\square$ & & 2010 & 2011 & 2012 & 2013 & 2014 & 2015 & 2016 & 2017 & 2018 & 2019 & $\begin{array}{r}\text { Annual \% change } \\
(95 \% \mathrm{CI}) \text { in the } \\
\% \mathrm{MRSA}\end{array}$ & $\begin{array}{r}p \\
\text { value }\end{array}$ \\
\hline \multirow[b]{3}{*}{ Japan } & S. aureus & 175,145 & 210,382 & 221,239 & 231,909 & 246,030 & 349,743 & 372,787 & 383,006 & 391,316 & 400,094 & \multirow[b]{3}{*}{$\begin{array}{r}0.94 \\
(0.92 \text { to } 0.96)\end{array}$} & \multirow[b]{3}{*}{$<0.01$} \\
\hline & MRSA & 100,845 & 114,933 & 117,209 & 118,539 & 120,702 & 169,528 & 177,768 & 182,619 & 185,709 & 192,320 & & \\
\hline & $\begin{array}{l}\% \text { MRSA ( } 95 \% \\
\mathrm{CI})\end{array}$ & $\begin{array}{r}57.6 \\
(57.4 \text { to } \\
57.8)\end{array}$ & $\begin{array}{r}54.6 \\
(54.4 \text { to } \\
54.8)\end{array}$ & $\begin{array}{r}53.0 \\
(52.8 \text { to } \\
53.2)\end{array}$ & $\begin{array}{r}51.1 \\
(50.9 \text { to } \\
51.3)\end{array}$ & $\begin{array}{r}49.1 \\
(48.9 \text { to } \\
49.3)\end{array}$ & $\begin{array}{r}48.5 \\
(48.3 \text { to } \\
48.7)\end{array}$ & $\begin{array}{r}47.7 \\
(47.5 \text { to } \\
47.9)\end{array}$ & $\begin{array}{r}47.7 \\
(47.5 \text { to } \\
47.9)\end{array}$ & $\begin{array}{r}47.5 \\
(47.3 \text { to } \\
47.7)\end{array}$ & $\begin{array}{r}48.1 \\
(47.9 \text { to } \\
48.3)\end{array}$ & & \\
\hline \multirow[b]{3}{*}{ Hokkaido } & S. aureus & - & - & - & - & - & 16,793 & 17,848 & 19,179 & 19,724 & 21,547 & \multirow[b]{3}{*}{$\begin{array}{r}0.26 \\
(0.04 \text { to } 0.48)\end{array}$} & \multirow[b]{3}{*}{0.11} \\
\hline & MRSA & - & - & - & - & - & 7,249 & 7,677 & 8,296 & 8,186 & 9,178 & & \\
\hline & $\begin{array}{l}\% \text { MRSA }(95 \% \\
\text { CI) }\end{array}$ & - & - & - & - & - & $\begin{array}{r}43.2 \\
(42.5 \text { to } \\
44.0)\end{array}$ & $\begin{array}{r}43 \\
(42.3 \text { to } \\
43.7)\end{array}$ & $\begin{array}{r}43.3 \\
(42.6 \text { to } \\
44.0)\end{array}$ & $\begin{array}{r}41.5 \\
(40.8 \text { to } \\
42.2)\end{array}$ & $\begin{array}{r}42.6 \\
(41.9 \text { to } \\
43.3)\end{array}$ & & \\
\hline \multirow{3}{*}{$\begin{array}{c}\text { JR } \\
\text { Sapporo } \\
\text { Hospital }\end{array}$} & S. aureus & 122 & 122 & 114 & 91 & 111 & 97 & 86 & 86 & 122 & 93 & \multirow[b]{3}{*}{$\begin{array}{r}1.43 \\
(0.42 \text { to } 2.43)\end{array}$} & \multirow[b]{3}{*}{0.05} \\
\hline & MRSA & 75 & 85 & 75 & 62 & 56 & 57 & 45 & 49 & 72 & 48 & & \\
\hline & $\begin{array}{l}\% \text { MRSA }(95 \% \\
\mathrm{Cl})\end{array}$ & $\begin{array}{r}61.5 \\
(52.6 \text { to } \\
69.7)\end{array}$ & $\begin{array}{r}69.7 \\
(61.0 \text { to } \\
77.2)\end{array}$ & $\begin{array}{r}65.8 \\
(56.7 \text { to } \\
73.9)\end{array}$ & $\begin{array}{r}68.1 \\
(58.0 \text { to } \\
76.8)\end{array}$ & $\begin{array}{r}50.5 \\
(41.3 \text { to } \\
59.6)\end{array}$ & $\begin{array}{r}58.8 \\
(48.9 \text { to } \\
68.1)\end{array}$ & $\begin{array}{r}52.3 \\
(41.9 \text { to } \\
62.5)\end{array}$ & $\begin{array}{r}57.0 \\
(4.5 \text { to } \\
66.9)\end{array}$ & $\begin{array}{r}59.0 \\
(50.1 \text { to } \\
67.3)\end{array}$ & $\begin{array}{r}51.6 \\
(41.6 \text { to } \\
61.5)\end{array}$ & & \\
\hline
\end{tabular}


3 The total numbers of $S$. aureus and MRSA isolates and the percentage of S. aureus isolates due to MRSA from 2010-19. The annual

4 percent change (the yearly rate of decline) in the percentage of $S$. aureus isolates due to MRSA; the $p$-value indicates whether the

5 time-dependent change is statistically significant. All datasets were from inpatient records. 


\section{Table 2 (on next page)}

Table 2. Annual percent increases in the proportion of methicillin-resistant Staphylococcus aureus (MRSA) susceptible to various drugs in JR Sapporo Hospital and across Japan from 2010-19.

Annual percent change (the yearly rate of decline); the $p$-value indicates the statistical significance of the time-dependent change. A likelihood ratio test was implemented, comparing a null model in which there is no time-dependent trend and an alternative model in which a linear trend was incorporated. NA, not available. 
1 Table 2. Annual percent increases in the proportion of methicillin-resistant Staphylococcus

2 aureus (MRSA) susceptible to various drugs in JR Sapporo Hospital and across Japan

3 from 2010-19.

\begin{tabular}{lllll}
\hline & \multicolumn{2}{c}{ MRSA (JR Sapporo Hospital) } & \multicolumn{2}{c}{ MRSA (Japan) } \\
\cline { 2 - 5 } & $\begin{array}{l}\text { Annual \% change } \\
(95 \% \mathrm{Cl}) \text { in cases }\end{array}$ & $p$ value & $\begin{array}{l}\text { Annual \% change } \\
(95 \% \mathrm{Cl}) \text { in cases }\end{array}$ & $p$ value \\
\hline Gentamicin & $\begin{array}{l}1.32(0.41 \text { to } 2.22) \\
-0.54(-0.98 \text { to }\end{array}$ & 0.045 & $2.51(1.45$ to 3.55) & $<0.01$ \\
Arbekacin & $-0.05)$ & NA & \\
Minocycline & $3.11(2.25$ to 3.94$)$ & $<0.01$ & $4.01(2.98$ to 5.01$)$ & $<0.01$ \\
Erythromycin & $0.90(0.50$ to 1.28$)$ & $<0.01$ & $0.79(0.11$ to 1.44$)$ & 0.11 \\
Clindamycin & $0.86(0.36$ to 1.34$)$ & 0.02 & $4.78(3.80$ to 5.71$)$ & $<0.01$ \\
Levofloxacin & $0.41(0$ to 0.82$)$ & 0.17 & $0.37(0.37$ to 1.10$)$ & 0.49 \\
Fosfomycin & $2.85(1.83$ to 3.85$)$ & $<0.01$ & NA & \\
Trimethoprim- & $-0.10(-0.25$ to & $<0.01$ & $0.01(-0.30$ to 0.10$)$ & 0.50 \\
sulfamethoxazole & $0.03)$ & & & \\
\hline
\end{tabular}

5 Annual percent change (the yearly rate of decline); the $p$-value indicates the statistical

6 significance of the time-dependent change. A likelihood ratio test was implemented, comparing a

7 null model in which there is no time-dependent trend and an alternative model in which a linear

8 trend was incorporated. NA, not available. 